\title{
Flavored Self Microemulsifying Lipid Formulations for Masking the Organoleptic Taste of Pharmaceutical Actives
}

\author{
Naser M Hasan*, Melfi S Al-aram, Mohammed S Al-wadie, Fahad A Althobaiti, Majed J Al-Malki \\ School of Pharmacy, Taif University, Saudi Arabia.
}

\begin{tabular}{|c|c|}
\hline ARTICLE INFO & ABSTRACT \\
\hline $\begin{array}{l}\text { Article history: } \\
\text { Received on: } 27 / 09 / 2015 \\
\text { Revised on: } 12 / 10 / 2015 \\
\text { Accepted on: } 29 / 10 / 2015 \\
\text { Available online: } 27 / 11 / 2015\end{array}$ & $\begin{array}{l}\text { The field of SMEDDS is designed to enhance bioavailability of poorly-water soluble compounds. Yet, these } \\
\text { systems have the capacity to solubilize aqueous-based materials within its lipid matrix as } \mathrm{L}_{2} \text { phase (W/O } \\
\text { microemulsion). This characteristic is utilized in this investigation to incorporate aqueous flavors within oil } \\
\text { vehicle as an approach to mask bitter taste of drugs. Miscibility profiles and self-micro-emulsifying regions for } \\
\text { various lipid composites were screened by constructing ternary phase diagrams using different types of oil, }\end{array}$ \\
\hline $\begin{array}{l}\text { Key words: } \\
\text { SMEDDS, Lipid } \\
\text { formulations, Micro- } \\
\text { emulsion, Taste masking, } \\
\text { Bitter taste drugs. }\end{array}$ & $\begin{array}{l}\text { Dynamic equilibrium phase studies were performed and phase boundaries were determined for the lipid-aqueous } \\
\text { flavors-water systems. Self-micro-emulsifying system comprising Crodamol GTCC/ Glycerox } 767 \mathrm{HC} / \text { Croduret } \\
40 \text { ss at ratios of }\{0 / 80 / 20\},\{6 / 54 / 40\} \text { or }\{10 / 40 / 50\} \text { have shown capacity to solubilize, aqueous-based materials } \\
\text { including; strawberry flavor, sucrose and citric acid as } \mathrm{L}_{2} \text { phase. Phase behavior study has revealed that clear } \\
\text { dispersions can be obtained at all dilutions with water. Potential flavored self-microemulsifying lipid } \\
\text { formulations representing type III lipid class system were developed. Aqueous flavors loaded into these vehicles } \\
\text { can be used to mask bitter tastes in oral pharmaceuticals. }\end{array}$ \\
\hline
\end{tabular}

\section{INTRODUCTION}

Self-micomulsifying drug delivery systems (SMEDDS) are isotropic mixtures of oils and non-ionic surfactants which spontaneously emulsify in water upon gentle agitation producing fine dispersions of particle size between 5 to $150 \mathrm{~nm}$. These systems exhibit one of the most successful approaches in improving the dissolution, absorption and hence bioavailability of poorly water-soluble compounds.

They provide a reservoir of drug dissolved in the lipid matrix, which upon administration and making contact with gastrointestinal fluids spontaneously emulsify producing oil-inwater dispersions with large surface area available for drug diffusion. However, In order to facilitate formulation design of lipid based formulations, these systems were classified into type I, II, III and IV (Pouton, 2006) based on the hydrophilicity of oil mixture, particle size of resultant dispersion and formulation digestibility (Table 1). Taste is crucial to oral pharmaceutical dosage forms as undesirable taste is one of the important formulation problems encounters many drugs.

* Corresponding Author

Email:naser_hasan@yahoo.com
Taste is the conscious experience produced when a ligand binds to its taste receptor cell (TRC) housed in the taste buds on different areas of the tongue. There are approximately 2000-5000 taste buds in the oral cavity (Miller, 1995). Each contains around 100 heterogeneous taste receptor cells (TRCs) and thus responds to all stimuli (Chandrashekar et al., 2006). Currently, there are five accepted taste primaries: salty, sweet, sour, bitter, and umami. Bitter or obnoxious taste of drugs leads to lack of patients' compliance and therefore, bitterness masking becomes essential. There are various known taste masking technologies which are used in pharmaceutical industry which include; addition of sweeteners and flavoring agents (Kalaskar and Singh, 2014), microencapsulation (Sharma and lewis, 2010; Alkire et al., 1997), granulation (Wadhwa and Puri, 2011; Ishikawa and Watanbe, 1999), pro-drug (Kalaskar and Singh, 2014), viscosity enhancers (Deepthi et al., 2011; Skraanga and Tully, 2000), pH modifiers (Deepthi et al., 2011), inclusion complexation (Leea et al., 2010), ion exchange resins (Puttewara et al., 2010), multiple emulsions (Khan et al., 2006) and the use of bitter taste inhibiting agents such as; neodesmin, aryl urea sulfonic acids (Roy et al., 1991), adenosine 5-monophosphate (ATP) (McGregor, 2004) and organic acids. 
Table 1: Typical Properties of Type I, II, III and IV Lipid Formulations (Pouton, 2006).

\begin{tabular}{|c|c|c|c|c|c|}
\hline & \multicolumn{5}{|c|}{ Increasing Hydrophilic Content } \\
\hline & Type I & Type II & Type IIIA & Type IIIB & Type IV \\
\hline $\begin{array}{c}\text { Typical Composition }(\%) \\
\text { Triglycerides or } \\
\text { Mixed Glycerides } \\
\end{array}$ & 100 & $40-80$ & $40-80$ & $<20$ & 0 \\
\hline Surfactants & 0 & $\begin{array}{c}20-60 \\
\text { HLB }<12\end{array}$ & $\begin{array}{c}20-40 \\
\mathrm{HLB}>12\end{array}$ & $\begin{array}{c}20-50 \\
\text { HLB }>12\end{array}$ & $\begin{array}{c}0-20 \text { HLB }<12 \\
20-80 \text { HLB }>12\end{array}$ \\
\hline $\begin{array}{c}\begin{array}{c}\text { Hydrophilic } \\
\text { Cosolvents }\end{array} \\
\end{array}$ & 0 & 0 & $0-40$ & $20-50$ & $0-80$ \\
\hline Particle Size of Dispersion (nm) & Coarse & $100-250$ & $100-250$ & $50-100$ & $<50$ \\
\hline Significance of Aqueous Dilution & $\begin{array}{l}\text { Limited } \\
\text { Importance }\end{array}$ & $\begin{array}{l}\text { Solvent Capacity } \\
\text { Unaffected }\end{array}$ & $\begin{array}{l}\text { Some Loss of Solvent } \\
\text { Capacity }\end{array}$ & $\begin{array}{c}\text { Significant Phase } \\
\text { Change and Potential } \\
\text { Loss of Solvent } \\
\text { Capacity }\end{array}$ & $\begin{array}{c}\text { Significant Phase } \\
\text { Change and Potential } \\
\text { Loss of Solvent } \\
\text { Capacity }\end{array}$ \\
\hline Significance of Digestibility & $\begin{array}{c}\text { Crucial } \\
\text { requirement }\end{array}$ & $\begin{array}{l}\text { Not Crucial But Likely } \\
\text { to Occur }\end{array}$ & $\begin{array}{l}\text { Not Crucial But May be } \\
\text { Inhibited }\end{array}$ & $\begin{array}{l}\text { Not Required and } \\
\text { Unlikely to happen }\end{array}$ & $\begin{array}{l}\text { Not Required and } \\
\text { Unlikely to happen }\end{array}$ \\
\hline
\end{tabular}

Microemulsion application for taste masking is discussed in literature (Patel et al., 2006; Silva et al., 2012) yet; there is no single available product on the market which uses this technology. Our findings (un-published data) have demonstrated that oil-water interface for emulsion dispersions is not rigid enough to conceal unpleasant taste of drugs and hence provide adequate protection from activating taste receptor cells on the tongue. Hence, the objective of the present study is to design and develop stable flavored $\mathrm{o} / \mathrm{w}$ micro-emulsions comprising various oils, cosurfactants, oil/aqueous based flavors and non-ionic surfactants using Paracetamol as bitter-taste hydrophilic model drug.

\section{MATERIALS AND METHODS}

\section{Materials}

Crodamol GTCC (medium chain triglyceride), Crodamol PC (propylene glycol dicaprylate / caprate), Glycerox 767HC (PEG 6 caprylic/ capric glycerides) and Croduret 40ss (PEG 40 Hydrogenated Castor Oil) were all supplied by Croda as gift samples. Cremophor RH 40 (PEG 40 Hydrogenated Castor Oil) was obtained from BASF Corporation, Oleic acid (USP) PRSCodex purchased from Panreac, Spain and Peppermint oil by Hayman Germany. Aqueous based strawberry flavor food grade by Foster Clark Product LTD was purchased from the local market. Paracetamol 98\% was obtained from Aldrich Company. Ethanol absolute was obtained from Scharlau, Spain.

\section{Methods}

\section{Miscibility profiles of lipid mixtures}

Regions of mutual solubility of various lipid formulations comprising various oils (Cordamol GTCC, Peppermint oil and Oleic acid), co-surfactants (Glycerox 767HC, Crodamol PC) and surfactants including;Crodurate 40 ss and Cremophor RH40 were constructed using ternary phase diagrams. Formulations of 2 grams which represent various percentages of oils, co-surfactants and surfactants on the ternary phase diagrams were weighed in $20 \mathrm{ml}$ glass test tubes and then tops were wrapped with parafilm. Mixtures were placed in a water bath at $50{ }^{\circ} \mathrm{C}$ for 2 minutes before lipid components were thoroughly vortexed.
Mixtures were then kept for $24-48$ hours in an oven set up at $25^{\circ} \mathrm{C}$ before visual assessment. Mixtures which formed a continuous single phase were classified as miscible formulations. Samples that displayed two or more phases were described as immiscible systems.

\section{Self-Emulsification profiles of lipid mixtures}

Mixtures of the various oils, co-surfactants and surfactants were produced by accurately weighing ingredients into glass test tubes and then wrapped by cling film followed by votrexing. Test tubes were held at $50{ }^{\circ} \mathrm{C}$ in a thermostated water bath held for 2 minute before lipid mixtures were thoroughly vortexed. Lipid formulations were then left to equilibrate over night in an oven set up at $25^{\circ} \mathrm{C}$. Emulsions were prepared under conditions of gentle agitation at a controlled temperature of $37^{\circ} \mathrm{C}$. An amount of $1 \mathrm{~g}$ of each lipid mixture was introduced into $100 \mathrm{ml}$ of distilled water in a $500-\mathrm{ml}$ glass beaker held at $37^{\circ} \mathrm{C}$ in a thermostated water bath. Emulsification under agitation conditions considered to be a reasonable simulation of the in vivo situation was carried out. Agitation was provided by gentle shaking on a mechanical shaker at 100 oscillations per min for 15 minutes. Visual assessment of resulting dispersions was carried out and systems which produced clear micro-emulsions were identified as SMEDDS.

\section{Phase behaviour study}

Selected lipid formulations with and without aqueous based strawberry flavor and Paracetamol were blended. Phase composition changes were made by adding water sequentially at $10 \% \mathrm{w} / \mathrm{w}$ intervals. Mixtures were vortexed until homogeneity is achieved and then allowed to stabilize for phase identification.

\section{Calibration curve of Paracetamol}

Calibration curve was constructed according to the method described by Pawar et al. (2013). An amount of $0.1 \mathrm{~g}$ of extracted Paracetamol was dissolved in $100 \mathrm{ml}$ ethanol. Series of dilutions were made to obtain Paracetamol concentrations ranging from $1 \mu \mathrm{g}$ to $10 \mu \mathrm{g}$. Absorbance of the various solutions were 
measured by UV spectrophotometry at $\lambda \max 250 \mathrm{~nm}$ using Jenway (Genova Model).

\section{Solubility of Paracetamol in various lipid mixtures}

Paracetamol is added in excess to various lipid solutions. Lipid suspensions are then vortexed for 3 minutes and then stored for 72 hours in a controlled temperature oven at $25^{\circ} \mathrm{C}$ to reach equilibrium (samples were vortexed in between). Oil suspensions are centrifuged at maximum speed $(10000 \mathrm{rpm})$ for 15 minutes. The clear saturated oil solution is then removed and assayed analytically by UV spectrophotometry at $\lambda \max 250 \mathrm{~nm}$.

\section{RESULTS AND DISCUSSION}

\section{Emulsification profiles of lipid formulations}

Lipid systems containing flavored nonpolar oil

The Emulsification profile of a lipid system composed of Oleic Acid(LCT oil), Peppermint Oil (Flavored Oil) and Cremophor RH40 (non-ionic surfactant) is shown in figure1. As figure 1 suggests, a very limited area of SMEDDS is obtained which reflects the optimized capacity of the surfactant to solubilize the non-polar content of the lipid mix, Oleic Acid and Peppermint Oil, in a stable $\mathrm{o} / \mathrm{w}$ microemulsion system after aqueous dispersion. At least $60 \%$ w/w of Cremophor RH40 is needed to be mixed with Peppermint Oil to produce SMEDDS. On the other hand, the maximum amount of Oleic acid to be included in the lipid mixture of Peppermint Oil/Cremophor RH40 and thus form SMEDDS is only about $10 \% \mathrm{w} / \mathrm{w}$ at minimum surfactant concentration of $70 \%$ w/w. Furthermore, replacing Oleic Acid (LCTs) with Crodamol PC (MCTs) has slightly extended SMEDDS area as depicted in figure 2 as generally, MCTs are more soluble and have higher mobility in the lipid/water interfaces. Optimized SMEDDS area can be obtained at maximum Oleic concentrations of $30 \% \mathrm{w} / \mathrm{w}$ in the lipidic mix w/w using minimum Cremophor RH40 concentrations of $60 \% \mathrm{w} / \mathrm{w}$.

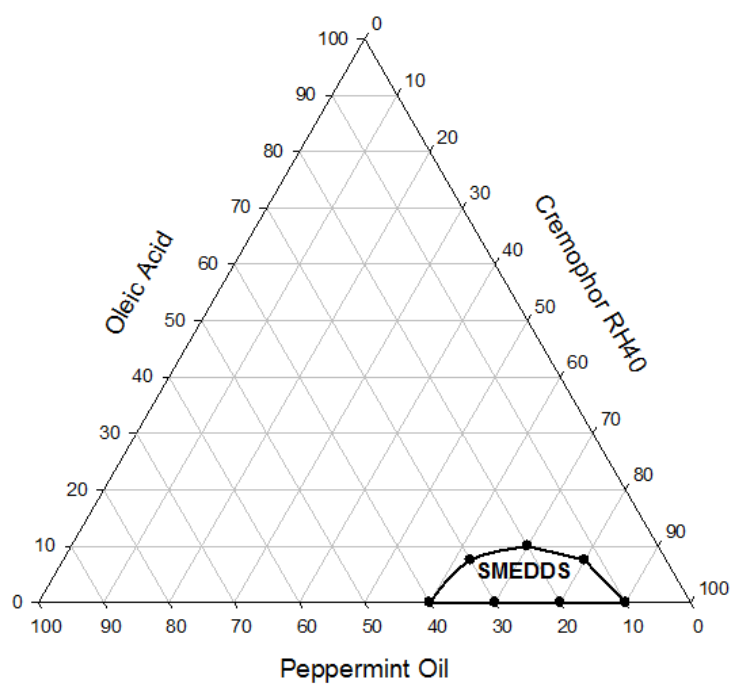

Fig. 1: Emulsification profile of a lipid system composed of Oleic Acid(LCTs oil), Peppermint Oil (Flavored Oil) and Cremophor RH40 (non-ionic surfactant). An amount of $1 \mathrm{~g}$ of representative single-phase lipid mixtures was emulsified in $100 \mathrm{ml}$ of water. Lipid systems which produced after emulsification in water optical clear dispersions were identified as SMEDDS.

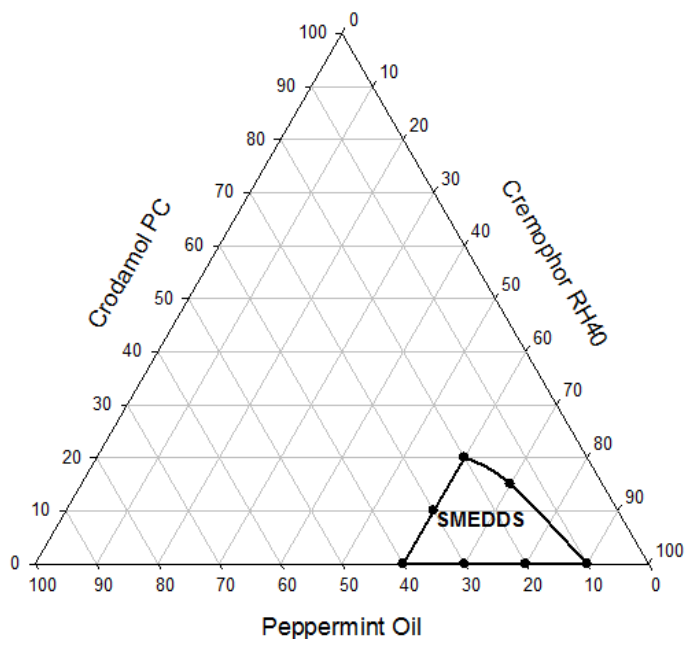

Fig. 2: Emulsification profile of a lipid system composed of Crodamol PC(MCTs oil), Peppermint Oil (Flavored Oil) and Cremophor RH40 (non-ionic surfactant). An amount of $1 \mathrm{~g}$ of representative single-phase lipid mixtures was emulsified in $100 \mathrm{ml}$ of water. Lipid systems which produced after emulsification in water optical clear dispersions were identified as SMEDDS.

\section{Lipid systems containing aqueous flavors}

Figure 3 shows the emulsification profile of a lipid system composed of Crodamol GTCC(oil), Glycerox 767HC (cosurfactant) and Croduret 40 ss (non-ionic surfactant). An extended area of SMEDDS is formed which widens the number of robust self-micro-emulsifying formulations that may be selected from this lipid composite. This may suggest the fact that due to the polar characteristics of Corduret 40 ss as measured by its HLB value (13) has, from one hand, improved the mixing properties of the lipid pre-concentrate and moreover with Glycerox 767HC (HLB = 13.2) have produced oil droplets with a total HLB value that would enhance degree of aqueous dispersion. Interestingly, self-microemulsifying dispersions can be obtained by blending a binary mix composed of Glycerox $767 \mathrm{HC}$ and Croduret 40 ss at only a $10 \%$ w/w minimum concentration of the non-ionic surfactant Croduret 40 ss, see line $\mathrm{AB}$ depicted on figure 3. Each of the lines $\mathrm{AC}, \mathrm{AD}$, $\mathrm{AE}$ and $\mathrm{AF}$ which are shown on figure 3 represents formulations at fixed ratio of Crodamol GTCC: Glycerox 767HC diluted with increasing concentration of Croduret 40 ss. Ratios of Crodamol GTCC: Glycerox $767 \mathrm{HC}$ for the representative formulations on lines $\mathrm{AC}, \mathrm{AD}, \mathrm{AE}$ and $\mathrm{AF}$ are 2:8, 3:7, 4:6 and 5:5, respectively. The progressive inclusion of Crodamol GTCC (source of triglyceride) in the lipid composite (i.e. moving from line $\mathrm{AC}$ to $\mathrm{AF}$ ) entails gradual increase of the concentration of the non-ionic surfactant Croduret 40 ss to produce SMEDDS. Based on the emulsification profile of Crodamol GTCC/Glycerox $767 \mathrm{HC} / \mathrm{Croduret} 40 \mathrm{ss}$ system, SMEDDS formulations numbered 1-12 presented in table 2 are selected. These formulations are categorized into group A; binary mix of Glycerox $767 \mathrm{HC} / \mathrm{Croduret}$ 40 ss using concentrations of Croduret 40 ss between $20-50 \%$ w/w, group B; binary mix of Crodamol GTCC/Glycerox 767HC at ratio of $\{1: 9\}$ blended with increasing concentrations of Croduret 
40 ss between $20-50 \% \mathrm{w} / \mathrm{w}$ and group $\mathbf{C}$; binary mix of Crodamol GTCC/Glycerox $767 \mathrm{HC}$ at ratio of $\{2: 8\}$ blended with increasing concentrations of Croduret 40 ss between $20-50 \%$ w/w. Furthermore, formulations numbered 13 to 19 in table 2 were selected based on emulsification behavior of lipid system composed of Crodamol PC, Oleic Acid and Crodurate 40 ss. Formulations 1-19 are considered pre-concentrate lipidic mixtures which will act as recipients to incorporating aqueous flavors within lipid matrix and hence producing as $\mathrm{W} / \mathrm{O}$ microemulsion system.

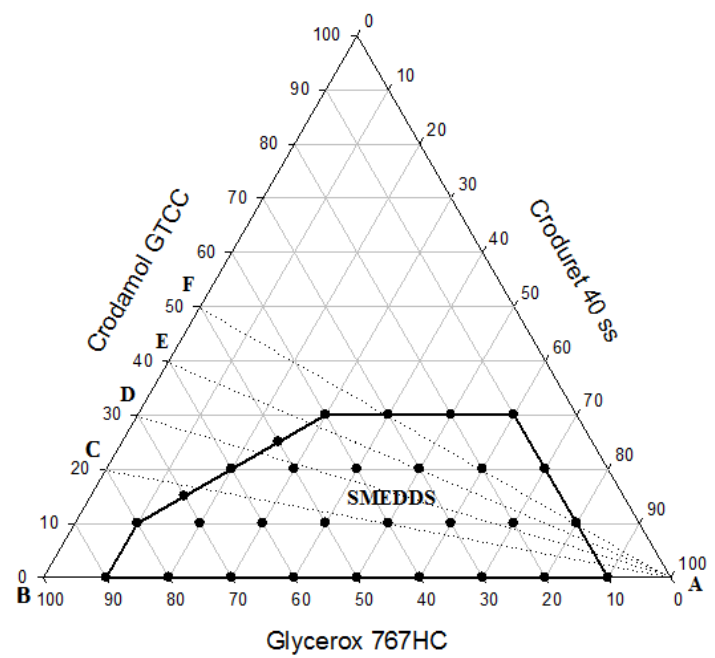

Fig. 3: Emulsification profile of a lipid system composed of Crodamol GTCC(MCT oil), Glycerox 767HC (co-surfactant) and Croduret40 ss (nonionic surfactant). An amount of $1 \mathrm{~g}$ of representative single-phase lipid mixtures was emulsified in $100 \mathrm{ml}$ of water. Lipid systems which produced after emulsification in water optical clear dispersions were identified as SMEDDS.

Table 2: Composition of the various SMEDDS formulation selected for loading aqueous based flavors as $\mathrm{O} / \mathrm{W}$ microemulsion.

\begin{tabular}{|c|c|c|c|c|c|}
\hline & \multicolumn{5}{|c|}{ Constituents \% (w/w) } \\
\hline Formulation & $\begin{array}{c}\text { Crodamol } \\
\text { GTCC }\end{array}$ & $\begin{array}{c}\text { Glycerox } \\
767 \mathrm{HC}\end{array}$ & $\begin{array}{c}\text { Crodamol } \\
\text { PC }\end{array}$ & $\begin{array}{l}\text { Oleic } \\
\text { Acid }\end{array}$ & $\begin{array}{c}\text { Croduret } \\
40 \text { ss }\end{array}$ \\
\hline 1 & 0 & 80 & 0 & 0 & 20 \\
\hline 2 & 0 & 70 & 0 & 0 & 30 \\
\hline 3 & 0 & 60 & 0 & 0 & 40 \\
\hline 4 & 0 & 50 & 0 & 0 & 50 \\
\hline 5 & 8 & 72 & 0 & 0 & 20 \\
\hline 6 & 7 & 63 & 0 & 0 & 30 \\
\hline 7 & 6 & 54 & 0 & 0 & 40 \\
\hline 8 & 5 & 45 & 0 & 0 & 50 \\
\hline 9 & 16 & 64 & 0 & 0 & 20 \\
\hline 10 & 14 & 56 & 0 & 0 & 30 \\
\hline 11 & 12 & 48 & 0 & 0 & 40 \\
\hline 12 & 10 & 40 & 0 & 0 & 50 \\
\hline 13 & 0 & 0 & 80 & 0 & 20 \\
\hline 14 & 0 & 0 & 70 & 0 & 30 \\
\hline 15 & 0 & 0 & 60 & 0 & 40 \\
\hline 16 & 0 & 0 & 50 & 0 & 50 \\
\hline 17 & 0 & 0 & 40 & 0 & 60 \\
\hline 18 & 0 & 0 & 4 & 36 & 60 \\
\hline 19 & 0 & 0 & 8 & 32 & 60 \\
\hline
\end{tabular}

\section{Phase behavior of aqueous-flavored lipid systems}

All 19 formulations presented in table 2 were titrated with increasing concentrations of aqueous-based food grade straw- berry flavor (Foster TM) from 0 to $20 \% \mathrm{w} / \mathrm{w}$ at $5 \%$ intervals. Dynamic phase behavior resulted from the interaction of increasing concentrations of strawberry flavor with the various lipidic formulations is shown in figure 4 . Formulations numbered 1-12 and 19 were able to solubilize $20 \%$ w/w of aqueous-based strawberry flavor forming stable w/o microemulsion identified as $\mathrm{L}_{2}$ phase. This reflects synergistic effect of Corduret 40 ss (HLB value $=13$ ) with Glycerox 767HC (HLB = 13.2) which have interplayed to forming the right HLB of the lipid mix to maximize solubilization of aqueous flavor into lipid matrix. Role of various lipid constituents on enhancement in the water-solubilized region $\left(\mathrm{L}_{2}\right)$ and hence, the impact on the mechanism of emulsification process is thoroughly investigated by Hasan (2014). Nonetheless, formulations from 13 to 18 have produced, on dilution with the aqueous flavor, a number dynamic intermediate transient phases passing from $\mathrm{L}_{2}$ phase $\rightarrow$ either $\mathrm{L}_{1}+\mathrm{L}_{2}$ (turbid mixtures) or gel phase. This will either compromise the stability of these systems or might unnecessarily extend emulsification time after aqueous dispersion of the formulation. These systems were deemed unsuccessful and hence were excluded from further evaluation.

Dynamic phase behavior of formulations 1 to 12 and 19 containing $20 \% \mathrm{w} / \mathrm{w}$ aqueous flavor resulted from the sequential dilution with water from $0-100 \% \mathrm{w} / \mathrm{w}$ was investigated and presented in figure 5. Formulations 1-4 (group A), 7 and 8 (group B) and 12 (group C) on dilution with water have produced all the way through clear $\mathrm{W} / \mathrm{O} \rightarrow \mathrm{O} / \mathrm{W}$ microemulsions which may suggest that these systems are good candidates to form the bases of producing flavored SMEDDS. On the other hand, formulations 5 and 6 (group B); 9, 10 and 11 (group C) and 19 have produced intermediate dynamic transient phases including $\mathrm{L}_{1}+\mathrm{L}_{2}$, Gel or $\mathrm{L}_{1}+\mathrm{L}_{2}+\mathrm{Gel}$ phases which may undermine the suitability of these systems and hence were excluded from further analysis.

Due to possible irritant effect of the non-ionic surfactant (Croduret $40 \mathrm{ss}$ ) present in these lipidic systems, formulations which have the minimal surfactant concentrations and still produce, on dilution with water, all the way through clear micromeulsions were selected for further development. Formulations which fulfill the aforementioned criteria are; formulation number 1 (group A) composed of Glycerox 767HC /Croduret 40 ss (80/20) \%w/w, formulation 7 (group B) composed of Crodamol GTCC/Glycerox 767HC /Croduret 40 ss (6/54/40) \% $\mathrm{w} / \mathrm{w}$ and formulation 12 (group C) composed of Crodamol GTCC/Glycerox 767HC /Croduret 40 ss (10/40/50) \%w/w.

Aqueous-based strawberry flavor containing $5-10 \% \mathrm{w} / \mathrm{w}$ sucrose was sequentially added to lipid formulations 1,7 or 12 . Dynamic phase behavior resulting from diluting lipidic formulations with increasing concentration of flavor containing sucrose is shown in figure 6 a. Clear W/O microemulsions as $\mathrm{L}_{2}$ phase were obtained at all added concentrations of flavor. This suggests that these formulations have high polarity to encapsulate enough hydrophilic sweetened flavors within the lipid matrix which can exert taste masking effect. Sucrose was used here as a natural sweetening agent. 


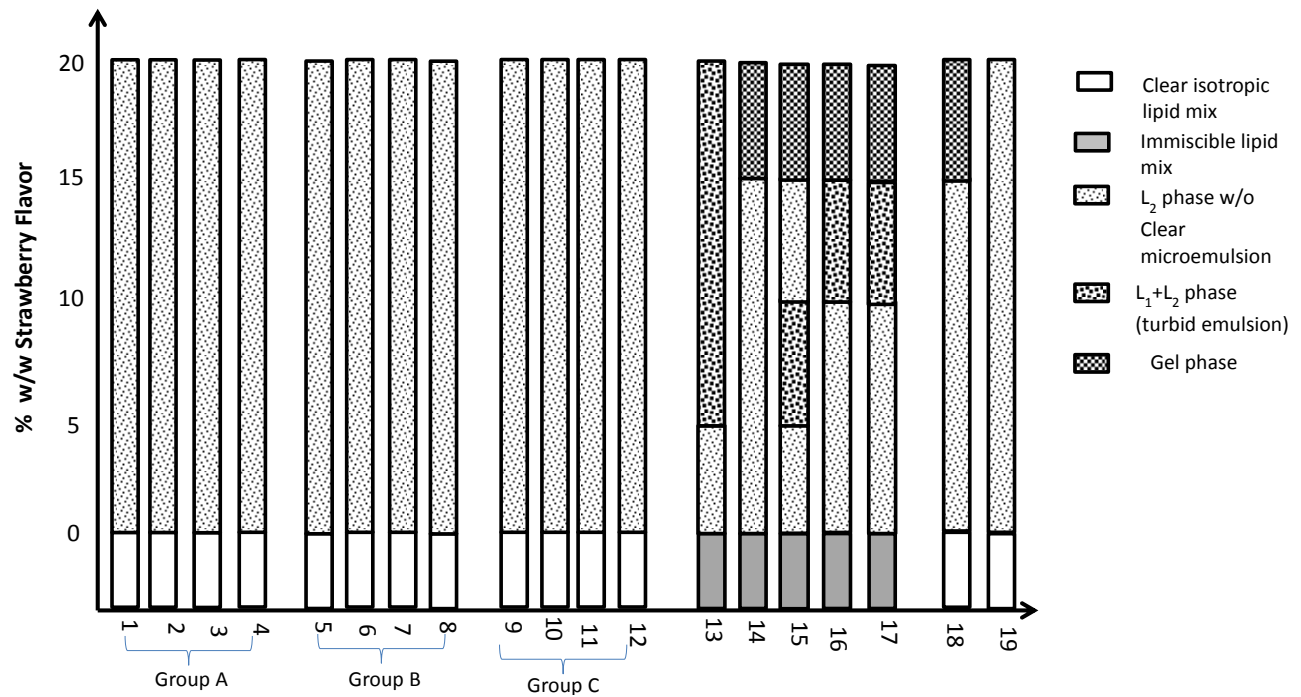

Fig. 4: Dynamic phase behavior of various lipidic formulations upon dilution with increasing concentrations of strawberry flavor.

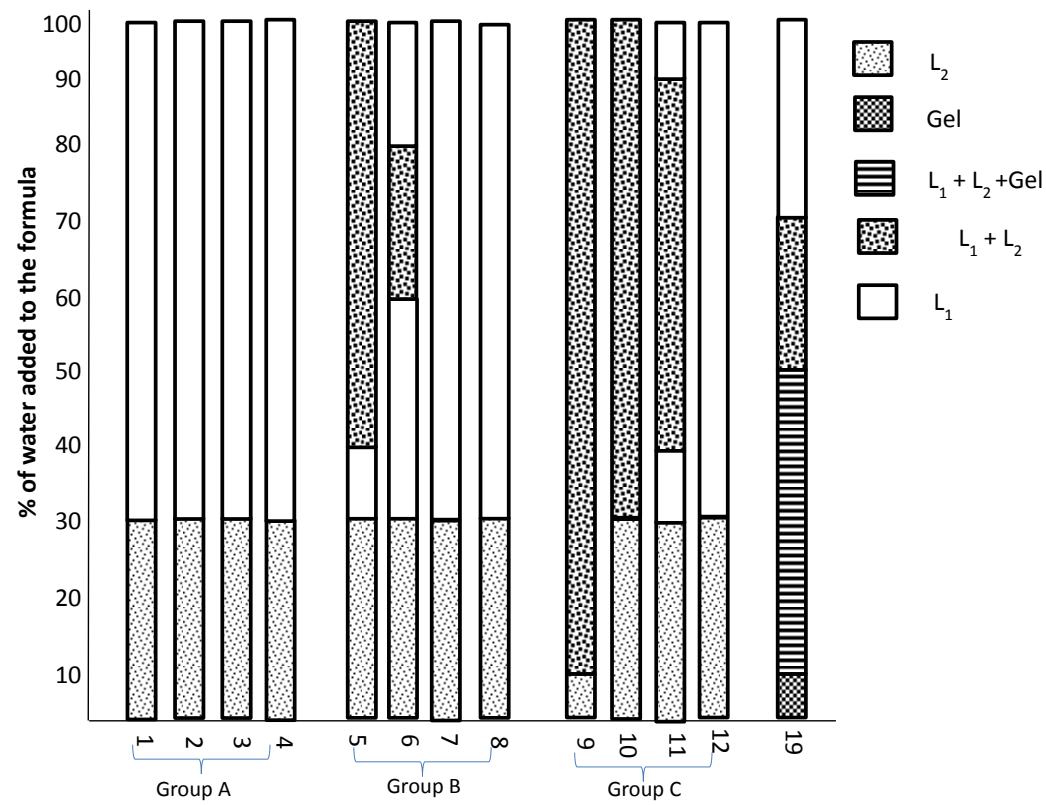

Fig. 5: Equilibrium phase behaviour for various lipid systems containing $20 \%$ (w/w) aqueous strawberry flavour on dilution with water.

However, synthetic sweeteners such as sucralose, aspartame and saccharin in combination sugar alcohols like lactitol, maltitol and sorbitol to decrease after-taste perception (Vummaneni and Nagpal, 2012) can also be used. Natural or synthetic flavors in combination with sweeteners can mimic taste masking efficiency. Strawberry flavor was here chosen as an example of aqueous-based flavor and cane by replaced by any other flavor according to the designed application.

Formulations 1,7 or 12 containing $20 \%$ w/w (strawberry flavor plus $5-10 \%$ sucrose) were further diluted with $0-100 \% \mathrm{w} / \mathrm{w}$ of water to highlight changes in the dynamic phases formed due to gradual penetration of water during emulsification process, see figure $6 \mathrm{~b}$. Clear $\mathrm{W} / \mathrm{O} \rightarrow \mathrm{O} / \mathrm{W}$ micromeulsions $\left(\mathrm{L}_{2} \rightarrow \mathrm{L}_{1}\right)$ are formed at all dilution percentages of water. This can improve patient's satisfaction and compliance as optical clear dispersions are obtained after dispersing formulation in any amount of water.
Lipidic formulations 1,7 and 12 are considered archetypical examples of type III lipid class systems according to the classification by Pouton (2006). Therefore, 'Diffusion and Stranding' mechanism is considered to be the predominant process of emulsification of these lipid systems. The role of co-surfactant (Glycerox 767HC) in these lipid mixtures is to aid in the emulsification process by the virtue of its high polarity. It is thought that co-surfactant can stabilize the interface by penetrating into the void spaces among surfactant molecules in the surfactant film around the oil droplet and hence lowering the interfacial tension and increasing the interfacial fluidity (Hasan, 2014).

Lipid formulations 1,7 and 12 were also titrated with increasing concentrations of strawberry flavor containing $10 \%$ $\mathrm{w} / \mathrm{w}$ sucrose and 5\% w/w citric acid. Equilibrium dynamic phase behavior has revealed clear W/O microemulsions $\left(\mathrm{L}_{2}\right.$ phase) which suggests that these lipid systems have the capacity to solubilize 
solution of sucrose and citric acid, see figure 7 a. Furthermore, on dilution with water of lipid systems containing $20 \% \mathrm{w} / \mathrm{w}$ (strawberry flavor, $10 \%$ sucrose and $5 \%$ citric acid), clear $\mathrm{W} / \mathrm{O} \rightarrow$ $\mathrm{O} / \mathrm{W}$ micromeulsions $\left(\mathrm{L}_{2} \rightarrow \mathrm{L}_{1}\right)$ are obtained, see figure $8 \mathrm{~b}$. Water soluble organic acids such astartaric acid, ascorbic acid or citric acid and their salts can be used with hydrophilic polymers, surfactant or oils for achieving taste masking. These acids promote salivation and thus viscosity in the mouth which can coat taste buds, and therefore can act as potential taste masking agent (Vummaneni and Nagpal, 2012). Furthermore, citric acid not only can increase taste masking efficiency of sweeteners but also can compete within the channel receptors with the bitter stimuli.

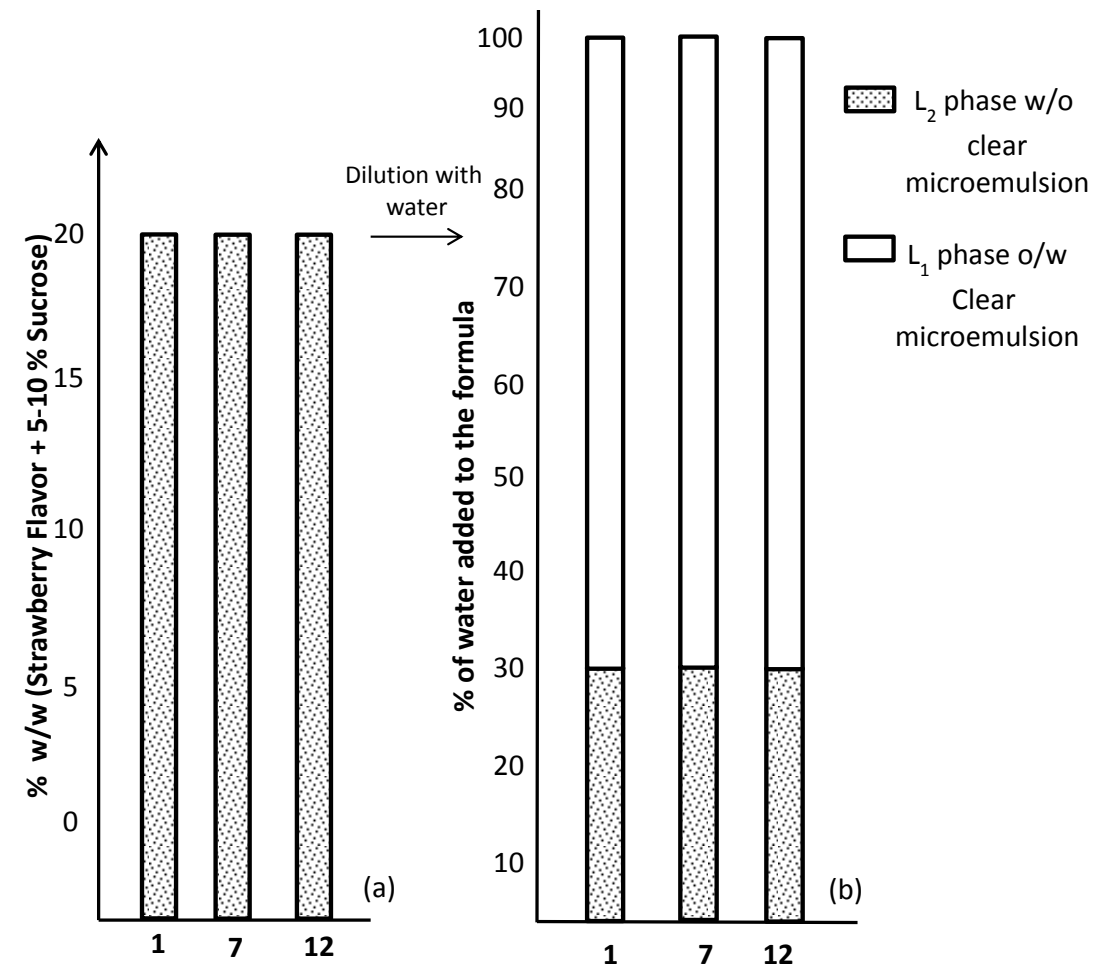

Fig. 6: Dynamic phase behavior resulting from diluting (a) Formulations 1, 7 or 12 with increasing concentration of aqueous-based strawberry flavor containing $5-10 \%$ w/w sucrose (b) Formulations 1,7 or 12 containing $20 \%$ w/w (strawberry flavor plus $5-10 \%$ sucrose) with $0-100 \%$ w/w of water.

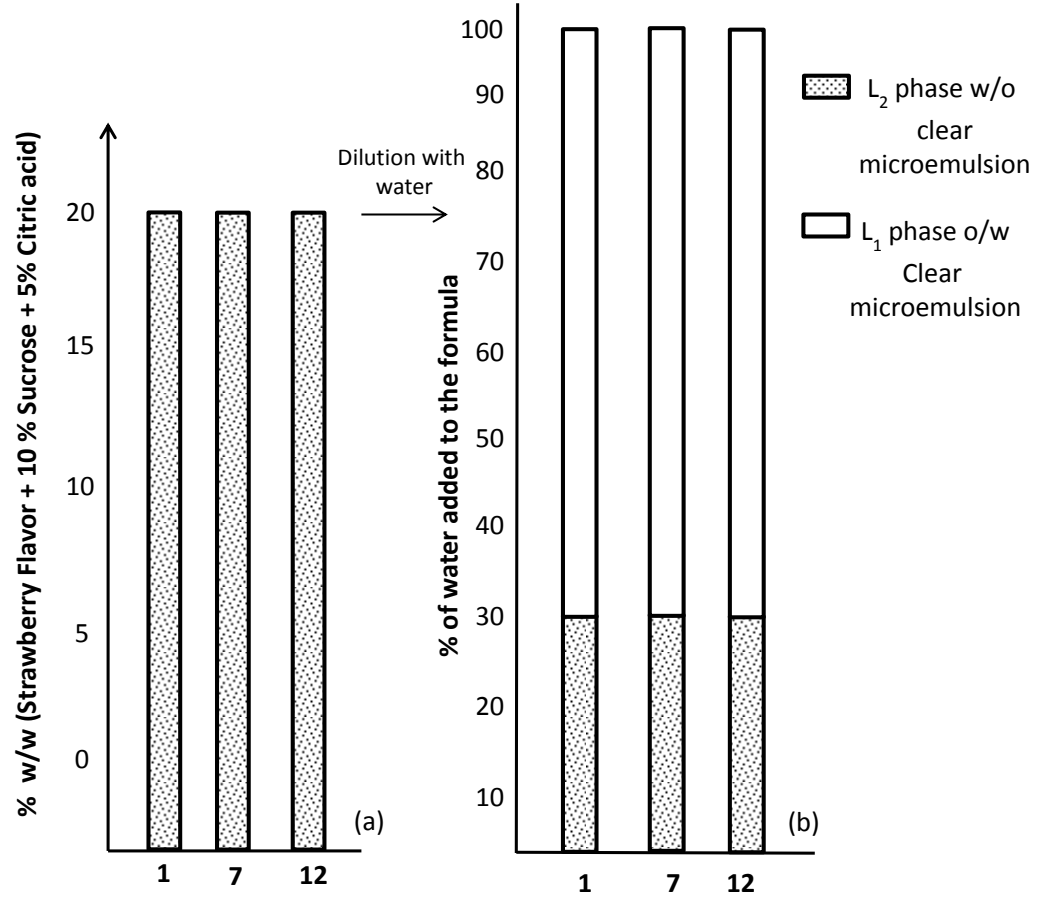

Fig. 7: Dynamic phase behavior resulting from diluting (a) Formulations 1, 7 or 12 with increasing concentration of aqueous-based strawberry flavor containing $10 \% \mathrm{w} / \mathrm{w}$ sucrose and 5\% w/w citric acid (b) Formulations 1, 7 or 12 containing $20 \% \mathrm{w} / \mathrm{w}$ (strawberry flavor, $10 \%$ sucrose and 5\% citric acid) with $0-100 \%$ w/w of water. 


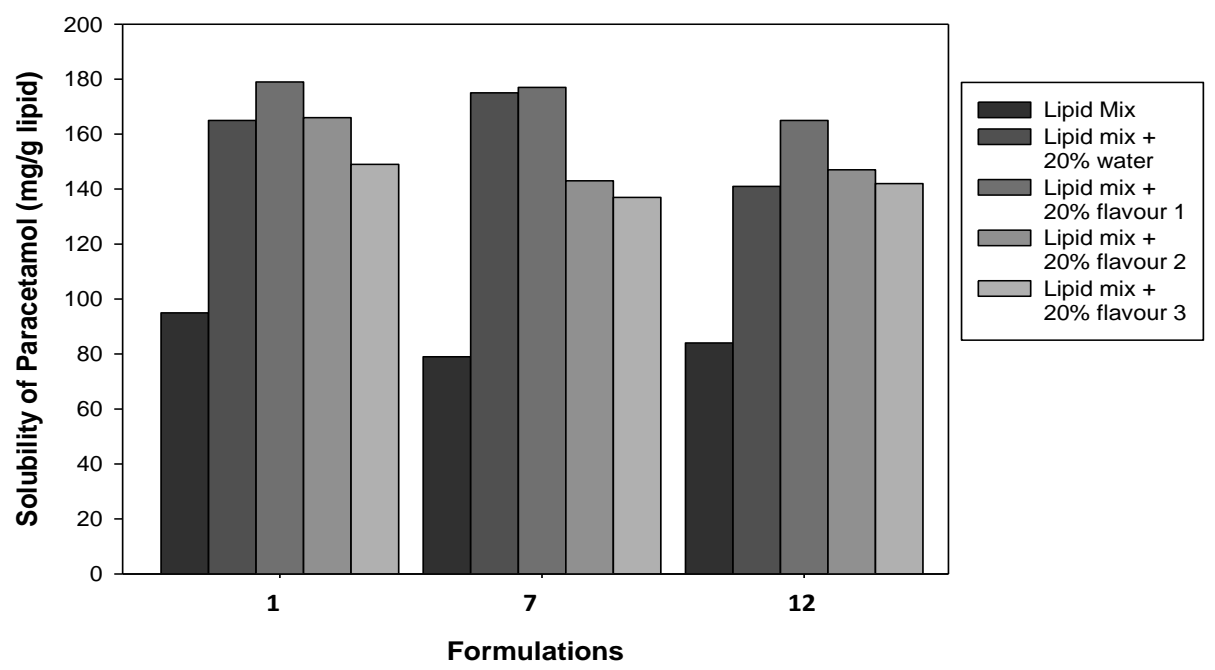

Fig. 8: Saturated solubility of Paracetamol in various lipidic solutions; flavor 1 (pure strawberry flavor), flavor 2 (strawberry fla vor $+10 \%$ sucrose) and flavor 3 (strawberry flavor $+10 \%$ Sucrose $+5 \%$ citric acid).

\section{Inclusion of a bitter-taste model drug}

Paracetamol is widely used as analgesic and antipyretic drug in all age groups; pediatric and geriatric patients. It was selected here as a model bitter-taste hydrophilic drug with a threshold concentration of $350 \mu \mathrm{g} / \mathrm{ml}$ (Cavallari et al., 2004; Sharma et al., 2012). Saturated solubility of Paracetamol in various lipidic solutions is measured and data is presented in figure 8 .

Solubility of Paracetamol in pure lipid formulations 1, 7 or 12 was found approximately in the range of 80 to $90 \mathrm{mg} / \mathrm{g}$ lipid; with the highest value observed in formulation 1 . However, when incorporating $20 \% \mathrm{w} / \mathrm{w}$ of either water or flavor 1 (pure strawberry flavor) in the lipid formulations, solubility of Paracetamol was almost doubled in comparison to the pure lipidic systems. Approximately 160 to $180 \mathrm{mg} / \mathrm{g}$ lipid is the solubility of Paracetamol in lipid formulations 1 or 7 containing $20 \% \mathrm{w} / \mathrm{w}$ of either water or flavor 1 . This is attributed to the fact that, as Paracetamol is a water soluble drug, increasing hydrophilic content within lipid matrix will ensue in enhancing solubility of Paracetamol in these systems. However, there is reduction in Paracetamol solubility in the case of including in the lipid systems, $20 \%$ of either flavor 2 (strawberry flavor $+10 \%$ sucrose) or flavor 3 (strawberry flavor $+10 \%$ Sucrose $+5 \%$ citric acid), relatively to formulations containing $20 \%$ of either water or pure strawberry flavor (flavor 1). This might be due to the competition of sucrose or citric acid solutes present in flavor 2 or 3 with Paracetamol within aqueous core of the lipidic solution. Nonetheless, solubility of Paracetamol in all lipid formulations containing $20 \% \mathrm{w} / \mathrm{w}$ of either flavors 2 or 3 lies between $140-160 \mathrm{mg} / \mathrm{g}$ lipid. It is worth noting here that, for the solubility data, it may be concluded that half of the solubilized Paracetamol is sequestered within the aqueous core of lipidic solution and the other half is in the oil phase. On the other hand, if required, co-solvents such as, propylene glycol, PEG or Ethanol can still be used to further enhance solubility Paracetamol in these lipidic vehicles.
It is important here to elucidate the effect of incorporating drug in the lipid matrix on the phase behavior after dispersion of the formulation. Generally, hydrophobic dugs included in SMEDDS affect dynamic mechanistic of emulsification process as transient intermediate phases might appear before reaching L1 phase (clear $\mathrm{O} / \mathrm{W}$ microemulsion).

This might be attributed to the effect of drug on the polarity of the oil droplet or by blocking charge transfer between molecules during emulsification. Yet, if this system were to be applied as a method to enhance oral bioavailability of poorly water soluble compounds, the formation of intermediate phases might help solubilization of drugs and thus avoiding crystallization of drugs during emulsification in the GIT. However, in the case of using SMEDDS as taste masking approach, the formation of such transient intermediate phases might compromise physical characteristics the resultant dispersions at certain percentage of water dilution. Therefore, it is important for these systems with and without drugs to form all the way through clear dispersion during dilution with water.

Equilibrium dynamic phase behavior resulting from the gradual dilution with water of lipid formulation 7 containing $20 \%$ w/w pure strawberry flavor and Paracetamol at concentrations of either $50 \mathrm{mg}$ or $100 \mathrm{mg} / \mathrm{g}$ lipid is presented in figure 9. Clear W/O $\rightarrow \mathrm{O} / \mathrm{W}$ micromeulsions $\left(\mathrm{L}_{2} \rightarrow \mathrm{L}_{1}\right)$ are obtained at both concentrations of the drug. This suggests that Paracetamol as a hydrophilic material does not interfere in the mechanistics of emulsification process and thus may add value to this system as a potential approach for taste masking.

Nonetheless, hydrophobic drugs can interfere in the mechanistic processes of emulsification by blocking charge movement through the system, via the direct complexation of the drug with some mixture components through its interaction with the liquid crystalline phase or by penetration into the surfactant interfacial monolayer. 


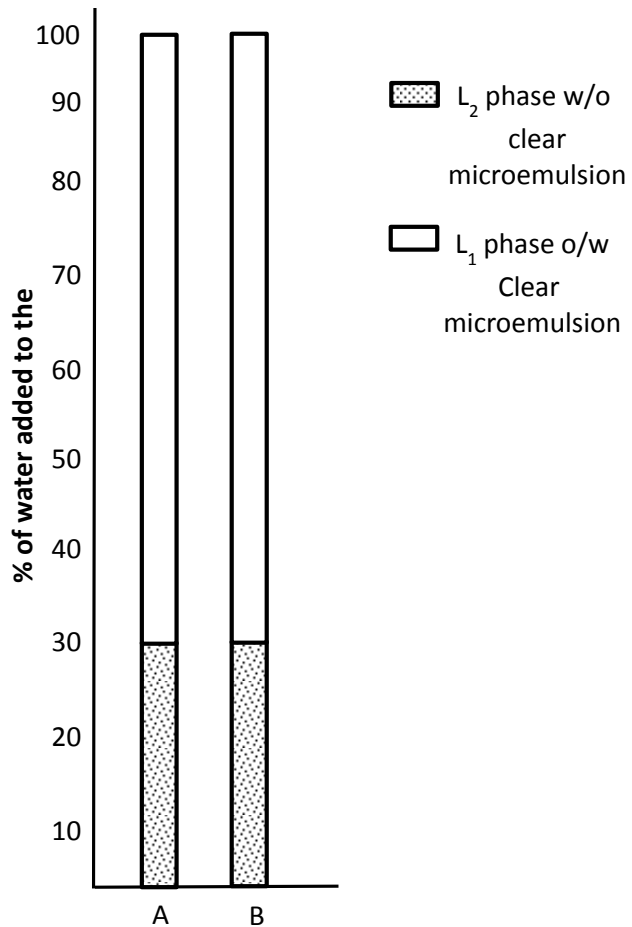

Fig. 9: Equilibrium dynamic phase behavior resulting from the gradual dilution with water of lipid formulation 7 containing $20 \%$ w/w pure strawberry flavor and Paracetamol at concentrations of (A) $50 \mathrm{mg}$ or (B) $100 \mathrm{mg} / \mathrm{g}$ lipid.

\section{CONCLUSION}

Potential flavored self-microemulsifying lipid formulations representing type III lipid class system which comprise Crodamol GTCC/ Glycerox 767HC /Croduret 40 ss at ratios of $\{0 / 80 / 20\},\{6 / 54 / 40\}$ or $\{10 / 40 / 50\}$ were developed. Aqueous-based strawberry flavor, sucrose, citric acid and Paracetamol could be loaded into these lipid systems forming stable $\mathrm{O} / \mathrm{W}$ microemulsion which can have taste masking abilities. These systems depending on formulation constituents can solubilize up to 140 to $180 \mathrm{mg}$ Paracetamol/gm lipid. Furthermore, clear microemulsions are obtained on further dilution with water, which adds value to these systems as potential taste masking vehicles.

\section{ACKNOWLEDGMENTS}

We are grateful to the Faculty of Pharmacy at Taif University for providing a good atmosphere and excellent facilities of research. I am also grateful to Crodaand BASF Corporation for providing excipients needed as gifts.

\section{REFERENCES}

Alkire TG, Sanftleben RA, Schuehle SS. Taste masking microparticles for oral dosage forms. U.S. Pat.No. 5,607,697 to Cima labs; 1997.

Cavallari C, Passerini N, Voinovich D, Gonzalez-Rodriguez ML, Megarotto L, Rodriguez L. Characterization and taste-masking evaluation of cetaminophen granules:comparison between different preparation methods in a high-shear mixer. Eur J Pharm Sci. 2004; 21:295-303.
Chandrashekar J, Hoon MA, Ryba NJP, Zuker CS. The receptors and cells for mammalian taste.Nature. 2006; 444, 288-294.

Deepthi P, Chowdary YA, Murthy GK, Seshagiri KB. Approaches for taste masking of bitter drugs. J Adv drug Res. 2011; 1(2):58-67.

Hasan NMY. Role of medium-chain fatty acids in the emulsification mechanistics of self-micro-emulsifying lipid formulations Saudi Pharm J. 2014; 22: 580-590.

Ishikawa T, Watanbe Y. Preparation and evaluation of tablets rapidly disintegrating in saliva containing bitter taste masked granules by the compression method. Chem Pharm Bull. 1999; 47: 1451-1454.

Kalaskar R, Singh RP. Taste masking: a novel technique for oral drug delivery system. Asian J Pharm Res Dev. 2014; 2(3):1-14.

Khan AY1, Talegaonkar S, Iqbal Z, Ahmed FJ, Khar RK. Multiple emulsions: an overview. Curr Drug Deliv. 2006; 3(4):429-43

Leea CW, Kima SJ, Yong-Suk Youna YS, et al. Preparation of bitter taste masked cetirizine dihydrochloride/ $\beta$-cyclodextrin inclusion complex by supercritical antisolvent (SAS) process.J Supercr Fluid. 2010; 55(1):348-357.

McGregor R. Taste modification in the biotech area. Food Tech. 2004; 58(5): 24-30.

Miller IJ. 1995. Anatomy of the peripheral taste system. In Handbook of Olfaction and Gustation. R.L. Doty, editor. Marcel Dekker, NY 521-547.

Patel N, Schmid U, Lawrence MJ.Phospholipid-based microemulsions suitable for use in foods. J Agric Food Chem. 2006; 54(20):7817-24

Pawar PY, Sandip H, Megha S, Arti A. Spectrophotometric estimation and validation of paracetamol and domperidone by different method from pure and tablet dosage form. Int J Drug Res Tech. 2013; 3 (2):37-44.

Pouton CW. Formulation of poorly water-soluble drugs for oral administration: physicochemical and physiological issues and the lipid formulation classification system. Euro J Pharm Sci. 2006; 29:278-87.

Puttewara TY, Kshirsagara MD, Chandewara AV, Chikhaleb RV. Formulation and evaluation of orodispersible tablet of taste masked doxylamine succinate using ion exchange resin. J king Saud Uni-Sci. 2010; 22(4): 229-240.

Roy G, Culberson C, Muller G, Nagarajan S. N-(sulfomethyl)$\mathrm{N}^{\prime}$-arylureas. US Patent 4994490; 1991.

Skraanga ATP, Tully RE. Oral liquid antidepressants solution.U.S. Patent 6,040,301; 2000.

Sharma D, Chopra R, Bedi N. Development and evaluation of paracetamol taste masked orally disintegrating tablets using polymer coating technique Int J Pharm Pharmac Sci. 2012; 4(3):129-133.

Sharma S, lewis S. Taste masking technologies: a review. Int J Pharm Pharmac Sci. 2010; 2(2):6-13.

Silva HD, Cerqueira MA, Vicente AA.Nanoemulsions for Food Applications: Development and Characterization. Food Bioprocess Technol. 2012; 5:854-867.

Vummaneni V, Nagpal D. Taste Masking Technologies: An Overview and Recent Updates. Int J Res Pharm Biomed. Sci. 2012; 3(2): 510-524.

Wadhwa J, Puri S. Taste masking: A novel approach for bitter and obnoxious drugs. Int J Biopharm and Tox Res. 2011; 1(1): 47-60.

\section{How to cite this article:}

Naser M Hasan, Melfi S Al-aram, Mohammed S Al-wadie, Fahad A Althobaiti, Majed J Al-Malki. Flavored Self Microemulsifying Lipid Formulations for Masking the Organoleptic Taste of Pharmaceutical Actives. J App Pharm Sci, 2015; 5 (11): 127-134. 